\title{
RADIAL VARIATION OF BLOCH FUNCTIONS
}

\author{
Peter W. Jones and Paul F. X. Müller
}

\section{The result}

In 1971 J. M. Anderson [A] conjectured that for any conformal map $\varphi$ in the unit disc there exists $\beta, 0 \leq \beta \leq 2 \pi$ such that

$$
\int_{0}^{1}\left|\varphi^{\prime \prime}\left(r e^{i \beta}\right)\right| d r<\infty
$$

More recently this problem has been posed in the works of N. Makarov [M], Ch. Pommerenke $[\mathrm{P}]$ and D. Gnuschke - Ch. Pommerenke $[\mathrm{G}-\mathrm{P}]$. The purpose of the present note is to prove Anderson's conjecture. This will be done by showing the following theorem about the associated Bloch function $b=\log \left|\varphi^{\prime}\right|$.

Theorem 1. There exists $\beta, 0 \leq \beta \leq 2 \pi$ such that

$$
b\left(r e^{i \beta}\right) \leq-\delta \int_{0}^{r}\left|\nabla b\left(\rho e^{i \beta}\right)\right| d \rho+\frac{1}{\delta}, \text { for } 0<r<1,
$$

where $\delta>0$ is independent of $r<1$.

The proof of Theorem 1 is given in section 3 where we also discuss how Anderson's conjecture follows.

\section{Preliminary inequalities}

In this section we recall three estimates due to J. Bourgain, Ch. Pommerenke and A. Beurling respectively. In section 2 the construction of stopping time Lipschitz domains is based on Pommerenke's inequality. In section 3 the selection of good directions $e^{i \beta}$ is based on the result of J. Bourgain and estimates for harmonic measure due to A. Beurling.

We first discuss Bourgain's inequality from [B]. For $e^{i \alpha} \in \mathbb{T}$ we let $\Gamma_{\alpha}$ be the collection of curves $\gamma$ which admit the following parametrization. For $0<r<1$, $\gamma(r)=r e^{i \alpha} e^{i \theta(r)}$ where $|\theta(r)|<C(1-r)$ and $\left|\theta^{\prime}(r)\right|<C$. We fix a non-negative, harmonic function $h$ in $\mathbb{D}$ and we let $K$ be an interval in $\mathbb{T}$. Then the following result was proven in $[\mathrm{B}]$.

Received January 20, 1997.

The first author is supported by N.S.F. Grant DMS-9423746.

The second author is supported by the Austrian Academy of Sciences, APART Program. 
Theorem 2. There exists $e^{i \alpha} \in K$ so that for each curve $\gamma \in \Gamma_{\alpha}$,

$$
\delta_{0} \int_{\gamma}|\nabla h|(\zeta)|d \zeta| \leq h(0)
$$

where $\delta_{0}>0$ depends only on $C$ and $|K|$.

The Bloch space $B$ consists of those harmonic functions $b: \mathbb{D} \rightarrow \mathbb{R}$ for which $\|b\|_{B}=\sup _{z \in \mathbb{D}}|\nabla b(z)|(1-|z|)$ is finite. Pommerenke's theorem (see [P, p. 78]) is the following.

Theorem 3. Let $b: \mathbb{D} \rightarrow \mathbb{R}$ be in the Bloch space $B$ and $\|b\|_{B} \leq 1$. Let $I \subseteq \mathbb{T}$ be an interval. Then there exists $e^{i \alpha} \in I$ so that for each $z \in\left\{r e^{i \alpha}: 0<r<1\right\}$, the estimate $|b(z)-b(0)| \leq 22|I|^{-1}$ holds.

For a conformal map $\varphi: \mathbb{D} \rightarrow \Omega$ the function $b=\log \left|\varphi^{\prime}\right|$ belongs to the Bloch space $B$ with Bloch norm $\leq 6$. This is a consequence of classical distortion theorems. ( See $[\mathrm{P}]$. ) Bloch functions are related to $\left|\varphi^{\prime \prime}\right|$ as follows. Let $g=$ $\log \varphi^{\prime}, g^{\prime}=\varphi^{\prime \prime} / \varphi^{\prime}$, hence $\left|\varphi^{\prime \prime}\right|=\left|g^{\prime}\right|\left|\varphi^{\prime}\right|$. Let $b=\log \left|\varphi^{\prime}\right|$, then $b$ is the real part of $g$. Hence by the Cauchy-Riemann equations $2\left|g^{\prime}\right|=|\nabla b|$, and

$$
2\left|\varphi^{\prime \prime}\right|=|\nabla b|\left|\varphi^{\prime}\right|=|\nabla b| e^{b} .
$$

This identity provides a link between estimates for the variation of Bloch functions and estimates for the $L^{1}$ norm of $\varphi^{\prime \prime}$. This has been exploited in [G-P].

Next we recall a minorization for harmonic measure due to A. Beurling. Fix $e \in \mathbb{D}$ and $0<\delta<1$. Then we define the Stolz angle $C(e, \delta)$ to be the convex hull of $\{z \in \mathbb{D}:|z|<(1-\delta)|e|\}$ and $e$. If $1 / 4<\delta<1$, then we write simply $C(e)$ for $C(e, \delta)$. Let $W \subseteq \mathbb{D}$ be a Lipschitz domain, $e \in \partial W$, and suppose that the cone $C(e, \delta / 2)$ is contained in $W$. Let $D$ be a connected subset of $\partial W$, so that $\operatorname{dist}(D, e)<r C_{1}$ and $\operatorname{diam}(D)>r C_{2}$. Then the following estimate holds.

Theorem 4. For each $z \in C(e, \delta)$, with $|e-z|<r$, the harmonic measure satisfies $\omega(z, D, W)>\eta_{0}$, where $\eta_{0}$ depends only on $C_{1}, C_{2}$, and $\delta$.

Below we will be concerned with the question whether a given curve in the unit disc remains in a fixed Stolz angle or not. To decide this the following criterion which is a folk theorem uses lower estimates for harmonic measure. We fix $e^{i \alpha} \in \mathbb{T}$, and let $I_{\alpha}=\left\{e^{i \theta}: \alpha<\theta<\alpha+\pi / 2\right\}$ and $J_{\alpha}=\left\{e^{i \theta}: \alpha-\pi / 2<\theta<\alpha\right\}$. For $z \in \mathbb{D}$ we denote by $\omega(z, I)$ the harmonic measure of $I$ with respect to $\mathbb{D}$ evaluated at $z \in \mathbb{D}$.

Theorem 5. For a path $\Gamma$ in $\mathbb{D}$ the following conditions are equivalent.

(i) There exists $\delta$ such that $\Gamma \subset C\left(e^{i \alpha}, \delta\right)$.

(ii) There exists $\eta>0$ such that for each $z \in \Gamma$, there hold the lower estimates for harmonic measure $\omega\left(z, I_{\alpha}\right)>\eta$ and $\omega\left(z, J_{\alpha}\right)>\eta$. 
We combine this criterion and Beurling's estimates. Let $f: \mathbb{D} \rightarrow W$ be the conformal map from the unit disc to the Lipschitz domain $W$. Fix $e \in \partial W$, and let $e^{i \alpha}=f^{-1}(e)$. Suppose that $C(e, \delta / 2) \subset W$, and let $\Gamma$ be a path in $C(e, \delta)$. Then the following holds.

Theorem 6. There exists $\delta_{0}>0$, depending only on $\delta$, such that $f^{-1}(\Gamma)$ is contained in $C\left(e^{i \alpha}, \delta_{0}\right)$.

\section{Stopping time Lipschitz domains}

In this section we define the stopping time Lipschitz domain $W\left(z_{0}\right)$, and we collect some of its basic properties. We let $b: \mathbb{D} \rightarrow \mathbb{R}$ be a Bloch function, and we fix it throughout this section. We also fix $z \in \mathbb{D}$ with $|z|>15 / 16$. First we construct an auxiliary domain $V(z)$.

Let $I=\{\zeta \in \mathbb{T}:|z-\zeta| \leq 8(1-|z|)\}$. The intervals $I_{1}, I_{2}$ have length $1-|z|$, they are attached to the left respectively right endpoint of $I$. Let $r_{1}=2|z|-1$, and let $S_{0}=\left\{w \in \mathbb{D}:|w|=r_{1}\right.$ and $\left.|w-z| \leq 1-|z|\right\}$. The left respectively right endpoint of $S_{0}$ are $s_{1}$ resp. $s_{0}$. By Theorem 3 there are line segments $S_{i}$ connecting $s_{i}$ to $I_{i}$ such that

$$
\left|b(w)-b\left(s_{i}\right)\right| \leq 25|| b|| / \omega\left(s_{i}, I_{i}\right),
$$

whenever $w \in S_{i}, i \in\{1,2\}$. We let $V(z)$ be the domain in $\mathbb{D}$ which is bounded by $S_{0} \cup S_{1} \cup S_{2}$.

The domain $V(z)$ satisfies

$$
\sup |b(w)-b(z)| \leq\|b\|_{B} / \omega_{0}
$$

where the supremum is taken over $S_{0} \cup S_{1} \cup S_{2}$, and where $\omega_{0}=\min \left\{\omega\left(s_{1}, I_{1}\right)\right.$, $\left.\omega\left(s_{2}, I_{2}\right)\right\} / 30$. The boundary of $V(z)$ intersects $\mathbb{T}$ in an interval $J$. For the harmonic measure of $J$ we have the lower estimate $w(z, J, V(z)) \geq 1 / 3$. Moreover we observe the following.

The angle formed by $S_{1}$ and $J$, resp. $S_{2}$ and $J$ is less than $\pi / 5$.

Next fix $z_{0} \in \mathbb{D}$ with $\left|z_{0}\right| \geq 15 / 16$, and $M \in \mathbb{N}$ large enough. We now turn to the construction of $W\left(z_{0}\right)$. Using a stopping time $W\left(z_{0}\right)$ is obtained as a subdomain of $V\left(z_{0}\right)$. For an interval $I \subseteq \mathbb{T}$ we let $T(I)=\{w \in \mathbb{D}: w /|w| \in$ $I$ and $|I| / 2<1-|w|<|I|\}$. Now we let $\left\{I_{i}: i \in \mathbb{N}\right\}$ be the collection of maximal dyadic intervals with the property that there exists $z_{i} \in T\left(I_{i}\right)$ with

$$
b\left(z_{i}\right)-b\left(z_{0}\right) \leq-M .
$$

The stopping time Lipschitz domain is defined as

$$
W\left(z_{0}\right)=V\left(z_{0}\right) \backslash \bigcup_{i=1}^{\infty} V\left(z_{i}\right) .
$$


For $z_{0}=0$ we define the points $z_{i}$ using the stopping time condition (2.2). By (2.4), each point $z_{i}$ satisfying (2.2) must have modulus $\geq 15 / 16$. Hence $V\left(z_{i}\right)$ is well defined and we put $W(0)=\mathbb{D} \backslash \bigcup_{i=1}^{\infty} V\left(z_{i}\right)$.

The following list of remarks collects the basic properties of $W\left(z_{0}\right)$.

Remarks.

1. It follows from (2.1) that $W\left(z_{0}\right)$ is a Lipschitz domain with starcenter $z_{0}$. The Lipschitz constant is independent of $z_{0}$.

2. Suppose that $w \in \partial V\left(z_{i}\right)$ for some $i>0$, and suppose also that $|w|<1$. Then for $M$ large enough we have that

$$
-2 M<b(w)-b\left(z_{0}\right)<-M / 2 .
$$

3. For $M$ large enough we have $V\left(z_{i}\right) \subset V\left(z_{0}\right)$. Moreover the estimate $b\left(z_{i}\right)-$ $b\left(z_{0}\right)<-M$ implies that

$$
1-\left|z_{i}\right| \leq\left(1-\left|z_{0}\right|\right) / 16 .
$$

4. Let $I\left(z_{0}\right)=\left\{\zeta \in \mathbb{T}:\left|\zeta-z_{0} /\right| z_{0}|| \leq\left(1-\left|z_{0}\right|\right) / 4\right\}$. Pick $\zeta_{0} \in I\left(z_{0}\right)$. Let $R$ be the ray connecting 0 to $\zeta_{0}$, and let $L=R \backslash V\left(z_{0}\right)$. Then $L$ is contained in the Stolz angle $C\left(z_{0}\right)$.

5. Let $K\left(z_{0}\right)$ be the convex hull of $z_{0}$ and $I\left(z_{0}\right)$, and let $D=K\left(z_{0}\right) \cap \partial W\left(z_{0}\right)$, then by Theorem $4, \omega\left(z_{0}, D, W\left(z_{0}\right)\right)>\omega_{0}$, where $\omega_{0}>0$ is a universal constant.

\section{The selection of a good ray}

In this section we fix a conformal map $\varphi$ in the unit disc, and we will select $e^{i \beta} \in \mathbb{T}$ such that

$$
\int_{0}^{1}\left|\varphi^{\prime \prime}\right|\left(r e^{i \beta}\right) d r<\infty
$$

Let $b=\log \left|\varphi^{\prime}\right|$ be the Bloch function associated to the Riemann map $\varphi$. The ray $L=\left\{r e^{i \beta}: 0<r<1\right\}$ will be chosen so that on $L$ there are points $Q_{k}$ satisfying

$$
b\left(Q_{k}\right)-b\left(Q_{k-1}\right)<-M / 3
$$

and

$$
\int_{l_{k}}|\nabla b(\zeta)| d|\zeta| \leq C_{0} M
$$

where $l_{k}$ is the line segment connecting $Q_{k}$ and $Q_{k-1}$. Note that (3.2) and (3.3) imply Theorem 1 and (3.1). Indeed, summing (3.2) gives

$$
b\left(Q_{k}\right)<-k M / 3 .
$$

Using (3.3) we obtain from (3.4),

$$
b(\zeta)<-k M / 3+C_{0} M, \text { for } \zeta \in l_{k} .
$$


Clearly (3.3) and (3.5) imply the conclusion in Theorem 1. Now recall that $2\left|\varphi^{\prime \prime}\right|=|\nabla b| e^{b}$, together with (3.3) and (3.5) this identity gives the following estimate.

$$
\begin{aligned}
\int_{0}^{1}\left|\varphi^{\prime \prime}\left(r e^{i \beta}\right)\right| d r & =\int_{0}^{1}\left|\nabla b\left(r e^{i \beta}\right)\right| e^{b\left(r e^{i \beta}\right)} d r \leq \sum_{k=0}^{\infty} \int_{l_{k}}|\nabla b|(\zeta) e^{b(\zeta)}|d \zeta| \\
& \leq e^{C_{0} M} \sum_{k=0}^{\infty} e^{-k M / 3} \int_{l_{k}}|\nabla b(\zeta)||d \zeta| \leq C_{0} e^{C_{0} M} M .
\end{aligned}
$$

Now we begin the proof of Theorem 1. First we give an inductive definition of an auxiliary sequence of points $e_{k} \in \mathbb{D}$. Their limit will be the point $e^{i \beta}$ satisfying the required properties (3.2) and (3.3).

Fix $M \in \mathbb{N}$ large enough and assume $b(0)=0$. Let $W(0)$ be the stopping time Lipschitz domain constructed in section 2. Clearly we have $0 \in W(0)$. Let $f: \mathbb{D} \rightarrow W(0)$ be the Riemann map normalized such that $f(0)=0$.

We use the conformal map $f$ to pull back $b$ from $W(0)$ to the unit disc $\mathbb{D}$. The composition $h=b \circ f$ is harmonic and satisfies $h>-2 M$ in $\mathbb{D}$, and $h(0)=0$. By Bourgain's theorem there exists $e^{i \alpha} \in \mathbb{T}$ such that

$$
\delta_{0} \int_{\gamma}|\nabla h(\zeta)||d \zeta| \leq M, \text { for } \gamma \in \Gamma_{\alpha} .
$$

Now we let $e_{1}=f\left(e^{i \alpha}\right)$. We distinguish between the cases $\left|e_{1}\right|=1$ and $\left|e_{1}\right|<1$. If we have $\left|e_{1}\right|<1$, then by $(2.3)$ we have $b\left(e_{1}\right)<-M / 2$. If $\left|e_{1}\right|=1$, then we stop the construction of the points $\left\{e_{k}\right\}$.

Next we give the induction step in the construction of the points $\left\{e_{k}\right\}$. We are given $e_{1}, \ldots, e_{l}$, points in $\mathbb{D}$, so that $I\left(e_{k+1}\right) \subset I\left(e_{k}\right)$ and $\left|I\left(e_{k+1}\right)\right|<\left|I\left(e_{k}\right)\right| / 4$, for $1 \leq k \leq l-1$. Let $D=\partial W\left(e_{l}\right) \cap K\left(e_{l}\right)$. D is connected, and by Remark 5 in Section 2, for the harmonic measure we have the estimate $w\left(e_{l}, D, W\left(e_{l}\right)\right) \geq \omega_{0}$. Let $f: \mathbb{D} \rightarrow W\left(e_{l}\right)$ with $f(0)=e_{l}$ be the Riemann map for the domain $W\left(e_{l}\right)$, then $K=f^{-1}(D)$ is an interval and $|K| \geq \omega_{0}$.

Again, the conformal map $f$ is used to pull back $b$ from $W\left(e_{l}\right)$ to the unit disc. The composition $h=b \circ f-b \circ f(0)$ is harmonic and satisfies $h>-2 M$ in $\mathbb{D}$ and $h(0)=0$. By Bourgain's theorem there exists $e^{i \alpha} \in K$ such that

$$
\delta_{0} \int_{\gamma}|\nabla h(\zeta)||d \zeta| \leq M, \text { for } \gamma \in \Gamma_{\alpha}
$$

Let $e_{l+1}=f\left(e^{i \alpha}\right)$. As $e_{l+1}$ is a point in $D=\partial W\left(e_{l}\right) \cap K\left(e_{l}\right)$ it follows from (2.4) that

$$
I\left(e_{l+1}\right) \subset I\left(e_{l}\right) \text { and }\left|I\left(e_{l+1}\right)\right|<\left|I\left(e_{l}\right)\right| / 4 .
$$

If $\left|e_{l+1}\right|<1$, then by $(2.3)$,

$$
b\left(e_{l+1}\right)-b\left(e_{l}\right)<-M / 2 .
$$

Otherwise, i.e., when $\left|e_{l+1}\right|=1$ we stop the construction of the points $\left\{e_{k}\right\}$. 
Having completed the construction of the points $\left\{e_{k}\right\}$ we let $e^{i \beta}=\lim e_{k}$. By (3.7) the limit exists and lies in $\mathbb{T}$. Moreover it follows that $e^{i \beta}=\bigcap I\left(e_{k}\right)$. We let $L$ be the ray connecting 0 and $e^{i \beta}$. Notice that $L$ intersects the boundary $\partial W\left(e_{k}\right)$ at least once and at most twice. We let $Q_{k}$ be the point in $L \cap \partial W\left(e_{k}\right)$ that has the smaller modulus. If we let $l_{k}$ be the line segment connecting $Q_{k}$ and $Q_{k+1}$ then clearly $l_{k}$ coincides with $L \cap\left\{W\left(e_{k}\right) \backslash V\left(e_{k+1}\right)\right\}$. Moreover by Remark 4,

$$
l_{k} \text { is a subset of } C\left(e_{k+1}\right) \text {. }
$$

Now we wish to verify (3.2) and (3.3). We fix $k \in \mathbb{N}$ and let $f: \mathbb{D} \rightarrow W\left(e_{k}\right)$ be the conformal map that was used in the definition of $e_{k+1} \in \partial W\left(e_{k}\right)$. Then we had determined $\alpha$ by $f\left(e^{i \alpha}\right)=e_{k+1}$. Now (3.9) and Theorem 6 imply that the curve

$$
f^{-1}\left(l_{k}\right) \text { is contained in } C\left(e^{i \alpha}\right) .
$$

Moreover by the distortion theorem, the curve $f^{-1}\left(l_{k}\right)$ can be decomposed into say $F_{1} \cup \cdots \cup F_{m_{0}}$, with a universal $m_{0}$, so that each of the $F_{i}$ is contained in a curve $\gamma \in \Gamma_{\alpha}$. By (3.6) this implies that

$$
\int_{f^{-1}\left(l_{k}\right)}|\nabla h(\zeta)||d \zeta| \leq C_{0} M
$$

where $h=b \circ f-b \circ f\left(e_{k}\right)$. A change of variables gives the desired

$$
\int_{l_{k}}|\nabla b(\zeta)||d \zeta| \leq C_{0} M
$$

To obtain (3.2) from (3.8) we observe that $L$ hits the boundary $\partial W\left(e_{k}\right)$ near $e_{k}$. More precisely, the hyperbolic distance between $Q_{k}$ and $e_{k}$ is bounded independent of $k$. Hence from (3.8) we obtain

$$
b\left(Q_{k+1}\right)-b\left(Q_{k}\right)<-M / 3,
$$

provided that $M$ is large enough.

\section{References}

[A] J. M. Anderson, Category theorems for certain Banach spaces of analytic functions, J. Reine Angew. Math. 249 (1971), 83-91.

[B] J. Bourgain, Bounded variation of measure convolution, Mat. Zametki 54 (1993), $24-33$.

[G-P] D. Gnuschke and Ch. Pommerenke, On the radial limits of functions with Hadamard gaps, Michigan Math. J. 32 (1985), 21-31.

[M] N. G. Makarov, Probability methods in the theory of conformal mappings, Leningrad Math. J. 1 (1990), 1-56.

[P] Ch. Pommerenke, Boundary behaviour of conformal maps, Springer-Verlag, Berlin, Heidelberg, 1992.

Department of Mathematics, Yale University, New Haven, CT 06520

E-mail address: jones@math.yale.edu, muller@math.yale.edu 